\title{
A Review on OFDM: Concept, Scope \& its Applications
}

\author{
Manushree Bhardwaj ${ }^{1}$, Arun Gangwar ${ }^{2}$, Devendra Soni ${ }^{3}$ \\ Department of Electronics \& Instrumentation \\ Amrapali Institute Of Technology \& Sciences \\ Haldwani, Nainital,Uttarakhand \\ ${ }^{2}$ Assistant Professor \\ Department of Electronics \& Communication \\ Amrapali Institute Of Technology \& Sciences \\ Haldwani, Nainital,Uttarakhand \\ ${ }^{3}$ Assistant Professor \\ Department of Electronics \& Communication \\ Bhagwant University \\ Ajmer
}

\begin{abstract}
Orthogonal frequency division multiplexing (OFDM) is a special case of multicarrier transmission where a single DataStream is transmitted over a number of lower rate subcarriers. In July 1998, the IEEE standardization group decided to select OFDM as the basis for their new 5-GHz standard aiming a range of data stream from 6 up to 54 Mbps. This new standard is the first one to use OFDM in packet-based communications. In wireless communication, concept of parallel transmission of symbols is used to achieve high throughput and better transmission quality. Orthogonal Frequency Division Multiplexing (OFDM) is one of the techniques for parallel transmission. The idea of OFDM is to split the total transmission bandwidth into a number of orthogonal subcarriers in order to transmit the symbols using these subcarriers in parallel. In this paper we will discuss the basics of OFDM techniques, role of OFDM in this era, its benefits and losses and also some of its application.
\end{abstract}

Keywords: Orthogonal Frequency Division Multiplexing(OFDM) ,BER, ISI, PAPR , DVB, DAB

\section{INTRODUCTION}

With the increase of communications technology, the demand for higher data rate services such as multimedia, voice, and data over both wired and wireless links is also increased. New modulation schemes are required to transfer the large amount of data which existing techniques cannot support. These techniques must be able to provide high data rate, allowable Bit Error Rate (BER), and maximum delay. Orthogonal Frequency Division Multiplexing (OFDM) is one of them. OFDM has been used for Digital Audio Broadcasting (DAB) and Digital Video Broadcasting (DVB) in Europe, and for Asymmetric Digital Subscriber Line (ADSL) high data rate wired links. OFDM has also been standardized as the physical layer for the wireless networking standard 'HIPERLAN2' in Europe and as the IEEE 802.11a, g standard in the US, promising raw data rates of between 6 and 54Mbps .Orthogonal Frequency Division Multiplexing (OFDM) is a digital transmission Method developed to meet the increasing demand for higher data rates in communications which can be used in both wired and wireless environments.[1]

\section{A. What is OFDM?}

Orthogonal frequency division multiplexing (OFDM) is a widely used modulation and multiplexing technology, which has become the basis of many telecommunications standards including wireless local area networks (LANs), digital terrestrial television (DTT) and digital radio broadcasting in much of the world. . In the past, as well as in the present, the OFDM is referred in the literature as Multi-carrier, Multi-tone and Fourier Transform. The OFDM concept is based on spreading the data to be transmitted over a large number of carriers, each being modulated at a low rate. The carriers are made orthogonal to each other by appropriately choosing the frequency spacing between them. A multicarrier system, such as FDM (aka: Frequency Division Multiplexing), divides the total available bandwidth in the spectrum into sub-bands for multiple carriers to transmit in parallel.[2] It combines a large number of low data rate carriers to construct a composite high data rate communication system. Orthogonality gives the carriers a valid reason to be closely spaced with overlapping without ICI. [3] 


\section{B. Why OFDM?}

In contrast to conventional Frequency Division Multiplexing, the spectral overlapping among subcarriers are allowed in OFDM since orthogonality will ensure the subcarrier separation at the receiver, providing better spectral efficiency and the use of steep band pass filter was eliminated. OFDM transmission system offers possibilities for alleviating many of the problems encountered with single carrier systems. It has the advantage of spreading out a frequency selective fade over many symbols. This effectively randomizes burst errors caused by fading or impulse interference so that instead of several adjacent symbols being Completely destroyed, many symbols are only slightly distorted. This allows successful reconstruction of majority of them even without forward error correction. Because of dividing an entire signal bandwidth into many narrow subbands, the frequency response over individual subbands is relatively flat due to subband are smaller than coherence bandwidth of the channel. Thus, equalization is potentially simpler than in a single carrier system and even equalization may be avoided altogether if Differential encoding is implemented.

\section{Principle of OFDM}

In digital communications, information is expressed in the form of bits. The term symbol refers to a collection, in various sizes, of bits [4]. OFDM data are generated by taking symbols in the spectral space using M-PSK, QAM, etc, and convert the spectra to time domain by taking the Inverse Discrete Fourier Transform (IDFT). Since Inverse Fast Fourier Transform (IFFT) is more cost effective to implement, it is usually used instead [3]. The main features of a practical OFDM system are as follows:

- Some processing is done on the source data, such as coding for correcting errors, interleaving and mapping of bits onto symbols. An example of mapping used is QAM.

- The symbols are modulated onto orthogonal sub-carriers. This is done by using IFFT.

- Orthogonality is maintained during channel transmission. This can be achieved by adding a cyclic prefix to the OFDM frame to be sent. The cyclic prefix consists of the L last samples of the frame, which are copied and placed in the beginning of the frame. It must be longer than the channel impulse response.

- Synchronization: cyclic prefix can be used to detect the start of each frame. This is done by using the fact that the $\mathrm{L}$ first and last samples are the same and therefore correlated.

- Demodulation of the received signal by using FFT.

- Channel equalization: the channel can be estimated either by using a training sequence or sending known so-called pilot symbols at predefined sub-carriers.

- Decoding and de-interleaving.

\section{Basic OFDM system}

The OFDM signal generated by the system in Figure $1 \& 2$ is at baseband ; in order to generate a radio frequency (RF) signal at the desired transmit frequency filtering and mixing is required. OFDM allows for a high spectral efficiency as the carrier power and modulation scheme can be individually controlled for each carrier. However in broadcast systems these are fixed due to the one-way communication. The basic principle of OFDM is to split a high-rate datastream into a number of lower rate streams that are transmitted simultaneously over a number of subcarriers. The block diagram showing a simplified configuration for an OFDM transmitter and receiver is given in Fig.1 \& Fig. 2.

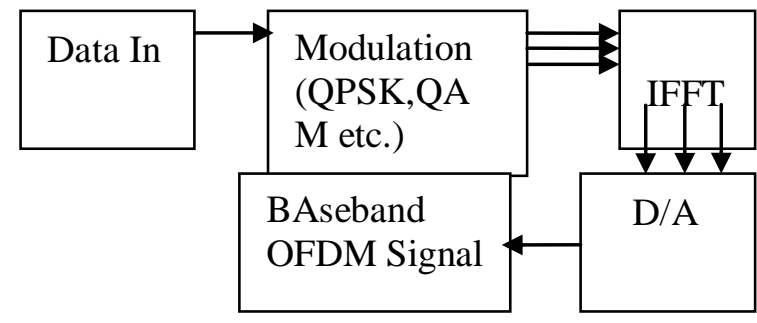

fig. 1: Transmitter 


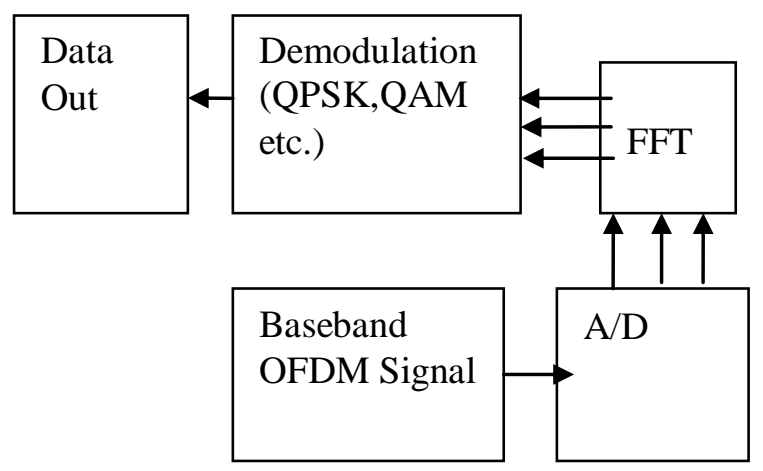

fig.2: Receiever

\section{E. OFDM Parameters and Characteristics}

The number of carriers in an OFDM system is not only limited by the available spectral bandwidth, but also by the IFFT size (the relationship is described by: number of carriers $\leq$ ((ifft - size $) / 2-2)$, which is determined by the complexity of thesystem [5]. The more complex (also more costly) the OFDM system is, the higher IFFT size it has; thus a higher number of carriers can be used, and higher data transmission rate achieved. The choice of M-PSK modulation varies the data rate and Bit Error Rate (BER). The higher order of PSK leads to larger symbol size, thus less number of symbols needed to be transmitted, and higher data rate is achieved. But this results in a higher BER since the range of 0-360 degrees of phases will be divided into more sub-regions, and the smaller size of sub-regions is required, thereby received phases have higher chances to be decoded incorrectly. OFDM signals have high peak-to-average ratio, therefore it has a relatively high tolerance of peak power clipping due to transmission limitations.

\section{F. Orthogonality}

The main aspect in OFDM is maintaining orthogonality of the carriers. If the integral of the product of two signals is zero over a time period, then these two signals are said to be orthogonal to each other. Two sinusoids with frequencies that are integer multiples of a common frequency can satisfy this criterion. Therefore, orthogonality is defined by:

$$
\int_{0}^{T} \cos \left(2 \pi n f_{o} t\right) \cos \left(2 \pi m f_{o} t\right) d t=0 \quad(n \neq m)
$$

Where $\mathrm{n}$ and $m$ are two unequal integers; $f_{\mathrm{o}}$ is the fundamental frequency; $T$ is the period over which the integration is taken. For OFDM, $T$ is one symbol period and $f_{0}$ set to $1 / T$ for optimal effectiveness [6and 7].

\section{ADVANTAGES OF OFDM:}

OFDM has several advantages over single carrier modulation systems and these make it a viable alternative for CDMA in future wireless networks. In this section, I will discuss some of these advantages.

- Multipath delay spread tolerance:

OFDM is highly immune to multipath delay spread that causes inter-symbol interference in wireless channels. Since the symbol duration is made larger (by converting a high data rate signal into N'low rate signals), the effect of delay spread is reduced by the same factor. Also by introducing the concepts of guard time and cyclic extension, the effects of inter-symbol interference (ISI) and inter-carrier interference (ICI) can be removed completely.

- Immunity to frequency selective fading channels:

If the channel undergoes frequency selective fading, then complex equalization techniques are required at the receiver for single carrier modulation techniques. But in the case of OFDM the available bandwidth is split among many orthogonal narrowly spaced sub-carriers. Thus the available channel bandwidth is converted into many narrow flat- fading sub-channels. Hence it can be assumed that the subcarriers experience flat fading only, though the channel gain/phase associated with the sub-carriers may vary. In the receiver, each sub-carrier just needs to be weighted according to the channel gain/phase encountered by it. Even if some sub-carriers are completely lost due to fading, proper coding and interleaving at the transmitter can recover the user data. 
- Efficient modulation and demodulation:

Modulation and Demodulation of the sub-carriers is done using IFFT and FFT methods respectively, which are computationally efficient. By performing the modulation and demodulation in the digital domain, the need for highly frequency stable oscillators is avoided. OFDM makes efficient use of the spectrum by allowing overlap.

- High transmission bitrates

- Chance to cancel any cannel if is affected by fading

- $\quad$ Flexibility: each transceiver has access to all subcarriers within a cell layer.

- Easy equalization: OFDM symbols are longer than the maximum delay spread resulting in flat fading channel which can be easily equalized.

- High spectral efficiency,

- $\quad$ Resiliency to RF interference.

- $\quad$ Lower multi-path distortion.

\section{DISADVANTAGES OF OFDM:}

- High synchronism accuracy.

- Multipath propagation must be avoided in other orthogonallity not be affected

- $\quad$ Large peak-to-mean power ratio due to the superposition of all subcarrier signals, this can become a distortion problem.

- $\quad$ More complex than single-carrier Modulation.

- $\quad$ Requires a more linear power amplifier.

- $\quad$ The OFDM signal has a noise like amplitude with a very large dynamic range, therefore it requires RF power amplifiers with a high peak to average power ratio.

- It is more sensitive to carrier frequency offset and drift than single carrier systems are due to leakage of the DFT.

- Peak to average power ratio (PAPR) is high.

- $\quad$ High power transmitter amplifiers need linearization.

- $\quad$ Low noise receiver amplifiers need large dynamic range.

- Capacity and power loss due to guard interval.

- Bandwidth and power loss due to the guard interval can be significant. 


\section{LIMITATIONS OF OFDM:}

There are some obstacles in using OFDM which are as given:

- OFDM signal exhibits very high Peak to Average Power Ratio (PAPR).

- Very sensitive to frequency errors (Tx. \& Rx. offset)

- Intercarrier Interference (ICI) between the subcarriers.

\section{OFDM APPLICATIONS:}

OFDM technique is the most prominent technique of this era .Some of its applications is given below.

- DAB: DAB - OFDM forms the basis for the Digital Audio Broadcasting (DAB) standard in the European market[8]. Digital Audio Broadcasting (DAB) using OFDM has been standardized in Europe [9] and is the next step in evolution beyond FM radio broadcasting providing interference free transmssion.

- HDTV

- Wireless LAN Networks

- $\quad$ 5.3.1 HIPERLAN/2

- $\quad$ IEEE $802.11 \mathrm{~g}$

- IEEE 802.16 Broadband Wireless Access System.

- Wireless ATM transmission system

- $\quad$ IEEE 802.11a

\section{CONCLUSION:}

The demand for high data rate wireless communication has been increasing drastically over the last decade. One way to transmit this high data rate information is to employ wellknown conventional singlecarrier systems.Since the transmission bandwidth is much larger than the coherence bandwidth of the channel, highly complex equalizers are needed at the receiver for accurately recovering the transmitted information. Multi-carrier techniques can solve this problem significantly. In this paper we have discussed about the basic idea behind the ofdm ,the most emerging technology of this era. Here we take a review on its concept, its properties in terms of its advantages and disadvantages ,its limitations and also its applications in different fields. This paper has explored the role of OFDM in the wireless communication and its advantages over single carrier transmission. There are also some limitations of this technique which can be removed with the help of suitabale techniques.

\section{REFERENCES:}

[1] G. Hill, M. Faulkner, and J. Singh, "Reducing the peak-to-average power ratio in OFDM by cyclically shifting partial transmit sequences," Electronics Letters, vol. 36, pp. 560-561, Mar 162000.

[2] Theory of Frequency Division Multiplexing: http://zone.ni.com/devzone/cda/ph/p/id/269

[3] Acosta, Guillermo. "OFDM Simulation Using MATLAB” 2000

[4] Litwin, Louis and Pugel, Michael. "The Principles of OFDM" 2001

[5] Tran, L.C. and Mertins, A. "Quasi-Orthogonal Space-Time-Frequency Codes in MB-OFDM UWB” 2007

[6] Understanding an OFDM transmission: http://www.dsplog.com/2008/02/03/understanding-an-ofdm-transmission/

[7] Minimum frequency spacing for having orthogonal sinusoidals http://www.dsplog.com/2007/12/31/minimum-frequencyspacing-for-having-orthogonal-sinusoidal

[8] G. Fay, "Wireless Data Networking," International Journal of Network Management, 8 March 1992, pp. 8-17.

[9] ETSI, "Radio Broadcast Systems: Digital Audio Broadcasting (DAB) to mobile, portable and fixed receivers," ETSI final draft ETS 300 401, Nov 1994. 